\title{
Modified double-patch technique for a combination of inferobasal postinfarct ventricular aneurysm and septal rupture
}

\author{
Arudo Hiraoka, MD, Toshinori Totsugawa, MD, Yusuke Irisawa, MD, and Hidenori Yoshitaka, MD
}

The patient was a 78-year-old woman who was diagnosed as having heart failure due to a combination of inferobasal postinfarct ventricular aneurysm and septal rupture (Figure 1, $A-C$ ).

The left ventricular (LV) aneurysm was opened at $1 \mathrm{~cm}$ lateral to the posterior descending artery (Figure 2, A). Pledgeted 3-0 polypropylene mattress sutures were placed all around the patch material and passed through the ventricular wall, avoiding the papillary muscle. At the side of the ventricular septum, the stitches were passed through the right ventricular wall from the more lateral side of the septum to exclude the ventricular defect (Figure 2, $A-C$ ). After BioGlue (CryoLife Inc; Kennesaw,
Ga) was delicately injected, the residual aneurysmal wall was closed, and a paired patch was tied down with the mattress sutures (Figure 2, D and E). The patient's postoperative course was uneventful, and the pulmonary to systemic perfusion ratio was improved from 3.61 to 1.12 (Figure 1, D-F).

In our modified technique, double-patch closure can achieve single-stage repair of inferobasal septal rupture and LV aneurysm. All transmural stitches and 4-fold closure (double patches, glue, and aneurysmal wall closure) reduced the risk of residual shunt and bleeding, and also reduced the pressure against the aneurysmal wall and outside patch.

\footnotetext{
From the Department of Cardiovascular Surgery, The Sakakibara Heart Institute of Okayama, Okayama, Japan.

Disclosures: Authors have nothing to disclose with regard to commercial support. Received for publication March 20, 2014; revisions received April 7, 2014; accepted for publication April 17, 2014; available ahead of print May 14, 2014.

Address for reprints: Arudo Hiraoka, MD, Department of Cardiovascular Surgery, The Sakakibara Heart Institute of Okayama, 2-5-1 Nakaicho, Kita-ku, Okayama 700-0804, Japan (E-mail: bassbord1028@yahoo.co.jp).

J Thorac Cardiovasc Surg 2014;148:2417-8 $0022-5223 / \$ 36.00$

Copyright (C) 2014 by The American Association for Thoracic Surgery http://dx.doi.org/10.1016/j.jtcvs.2014.04.033
}
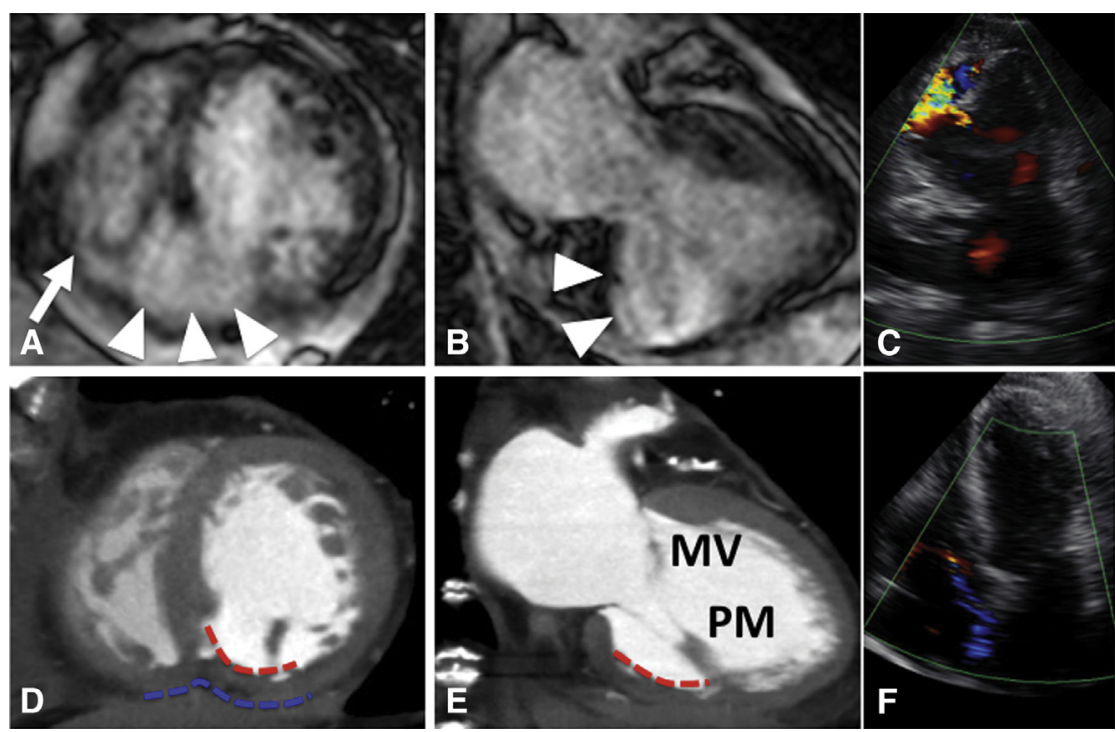

FIGURE 1. A and B, Preoperative cardiac magnetic resonance imaging showed inferobasal aneurysmal change (white arrowheads) and septal defect (white arrow) at the same lesion. C, Preoperative transthoracic echocardiography revealed a septal defect with a severe left-to-right shunt (diameter, $11.3 \mathrm{~mm}$ ). D and E, Postoperative cardiac computed tomography revealed no residual shunt and complete exclusion of the aneurysm. The red broken line and the blue broken line represent the inside and outside patches, respectively. $M V$, Mitral valve; $P M$, papillary muscle. F, Postoperative transthoracic echocardiography demonstrated the absence of a residual shunt. 

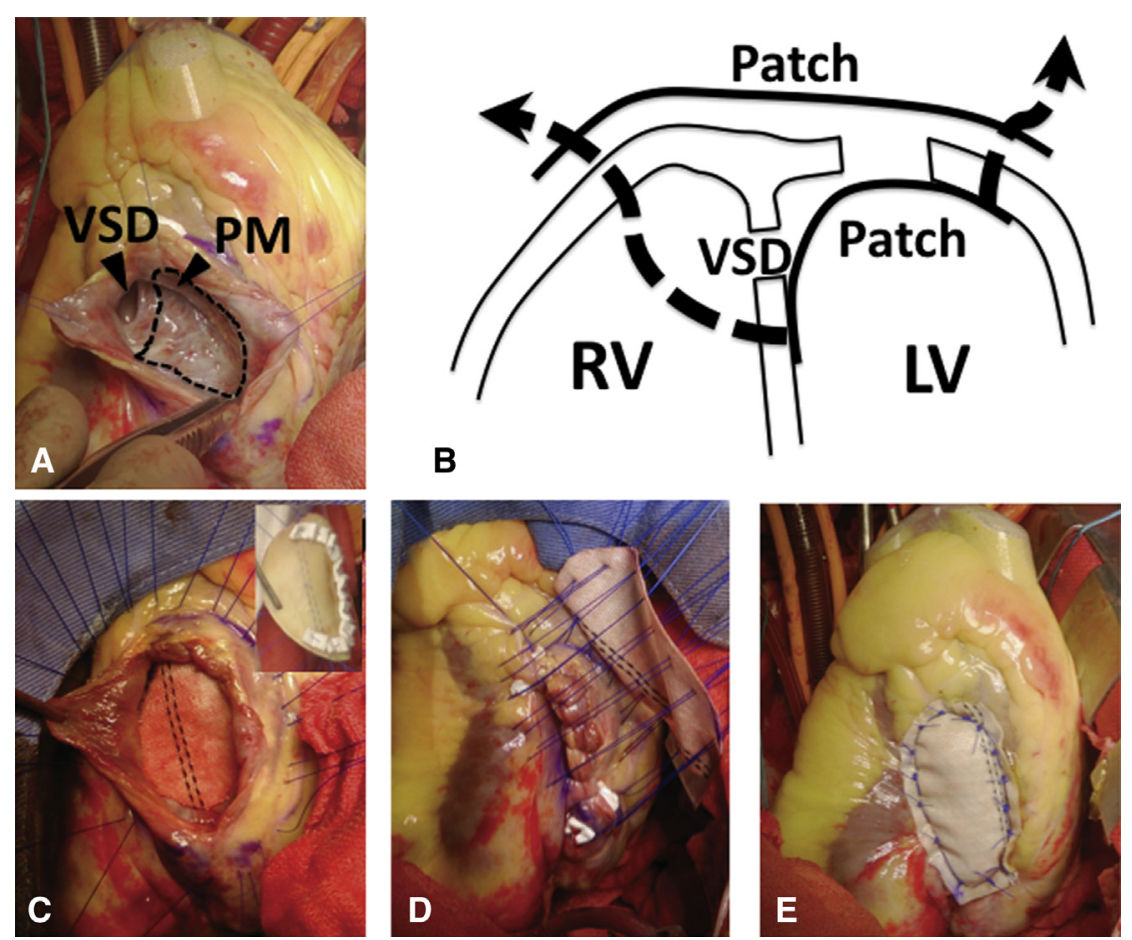

FIGURE 2. A, The inferior ventricular aneurysm was opened at $1 \mathrm{~cm}$ lateral to the posterior descending artery. The anatomic relationship between the ventricular septal defect $(V S D)$ and the papillary muscle $(P M)$ was easily verified. The black broken line indicates the line of mattress sutures. B, Schema of the technique. Double patches were placed with transmural stitches. At the side of ventricular septum, the stitches were passed through the right ventricular wall from more lateral side of the septum to exclude the ventricular septal defect (VSD). $R V$, Right ventricle; $L V$, left ventricle. C, A Xenomedica patch (Baxter Healthcare Corp, Horw, Switzerland) was attached to a Dacron polyester fabric patch (Hemashield; Maquet Cardiovascular, Wayne, NJ) with glue and elliptically trimmed $(4 \times 3 \mathrm{~cm})$. Pledgeted 3-0 polypropylene mattress sutures were placed all around the patch material and passed through the ventricular wall, avoiding the papillary muscle (Xenomedica patch faced to the left ventricular cavity). The initial patch was attached completely inside the left ventricle. D and E, The residual aneurysmal wall was closed, and a paired patch was tied down with the mattress stitches. 